\title{
SOCIAL STANCES BY VIRTUAL SMILES
}

\author{
Magalie Ochs, Catherine Pelachaud, and Ken Prepin
}

\author{
CNRS-LTCI \\ Télécom ParisTech \\ \{magalie.ochs,catherine.pelachaud,ken.prepin\}@telecom-paristech.fr
}

\begin{abstract}
When two persons participate to a discussion, they not only exchange about the concepts and ideas they are discussing, but they also express stances with regard to content of their speech (called epistemic stances) and to convey their interpersonal relationship (called interpersonal stances). The stances can be expressed through non-verbal behaviors, for instance smiles. Stances are also co-constructed by their interactants through simultaneous or sequential behaviors such as the alignment of speaker's and listener's smiles.

In this paper, we present several studies exploring the stances (epistemic, interpersonal, and co-constructed) that the social signal of smile may convey. We propose to analyze different contextual levels to highlight how users' engagement and discourse context influence their perception of the virtual characters' stances.
\end{abstract}

\section{INTRODUCTION}

During an interaction, people not only exchange the concepts and ideas which constitute the subject of their discussion, but they also express feelings, judgments or commitments regarding this subject and the relationship they entertain. This "attitude which, for some time, is expressed and sustained interactively in communication, in a unimodal or multi-modal manner" [1] corresponds to stance. Stances may refer to the relationship of a person with her own talk, called epistemic stance (for instance, “to be certain"); it may also convey information about the relationship of a person with her interlocutor, called interpersonal stance (for example "to be warm" or "to be polite") [2].

During human-machine interaction, a virtual character may be implied in different relationship with its interlocutor (be virtual or human) depending for instance on its embodied roles (such as tutor or game companion). Virtual characters should then have the capacity to express stances through their verbal and non-verbal behavior. One important social cue of stance during interaction is smile. Smile may convey stances

This work has been partially financed by the European Community Seventh Framework Program (FP7/2007-2013): the European Project VERVE and the European Project NoE SSPNet. to the perceiver both through the morphological and dynamic characteristics of the signal itself and through the context in which the smile is displayed. Indeed, a smile may express totally different stances - such as amusement or politeness depending on subtle characteristics of the signal. Moreover, the social signal of smile is "profoundly influential" [3]. The smiling behavior, i.e. when and which types of smiles are expressed during an interaction, may determine the perceived stances $[4,5]$.

In this article, we present studies on the stances that the social signal of smile may convey. We analyze the stances at different contextual levels:

- at a signal level, we attempt to highlight the relation between the morphological and dynamic characteristics of a smiling virtual face and the perceived stances (Section 2);

- at an utterance level, we consider the context in which virtual speakers delivering a verbal message display different smiles, and we analyze their effects on the user's perception of the virtual character's stances;

- at a communicative level, in which users converse in natural language with virtual characters. We study the effects of different virtual characters' smiling behaviors on the perceived stances (Section 4);

- at an interactive level, we show the impact of the reciprocal expressions of smile during an interaction on the perceived co-constructed stances (Section 5).

\section{SIGNAL LEVEL: DIFFERENT VIRTUAL SMILING FACES FOR DIFFERENT STANCES}

Virtual smiling faces, without any consideration of a particular context, may convey different interpersonal stances. A (genuine) smile is characterized by the activation of the zygomatic major muscles, on either side of the face. However, other muscles may be involved in an expression of smile. Depending on the activated muscles and how they are activated, different types of smile with different meanings can be distinguished [6]. The most common type of smile is the 
amused smile (also called felt or Duchenne smile). Another type, which is often thought of as the amused smile's opposite is the polite smile (also called non-Duchenne, false, social smile). A specific smile appears in the facial expression of embarrassment [6]. In this paper, we focus on the smiles of amusement and politeness.

In the domain of virtual agents, several existing virtual characters smile during an interaction, mainly to express a positive emotion or a positive mood. Only few researchers have considered different virtual agent's smiles. In most of the works, the amused smile is used to reflect an agent's emotional state of happiness whereas a polite smile is mainly considered as a particular smile masking an agent's negative emotion (e.g. [7]. The characteristics of the smiles are based on the theoretical descriptions of smiles proposed in [6]. However, even if the research shows that these different smiles are distinguishable by their distinct morphological and dynamic characteristics, no consensus exists on the morphological and dynamic characteristics of the amused and polite smiles in the literature in Human and Social Sciences [6, 8]

Based on a user-perception approach using a crowdsourcing method, we have developed a method to identify the morphological and dynamic characteristics of the amused and the polite smile of a virtual character [9]. We have created a web application that enables a user to easily create different types of smile on a virtual character's face by manipulating radio buttons on an interface to change the parameters of the smiles (such as the amplitude of smile, the mouth opening, the lip press, or the cheek raising). Based on a decision tree learning algorithm, the analysis of the corpus of virtual smiling faces created by users has enabled us to define, not only one single smile for each smile type, but several smiles of amusement and politeness. The resulting smiles have been validated as the most appropriate smile expressions in scenarios of amusement and politeness [9]. This study on the social signal of smile shows that the characteristics of the signal may convey particular stances at a signal level, such as amusement or politeness. However, a smile displayed by a virtual character may convey different stances depending, for instance, on the verbal message that comes with the social signal.

\section{UTTERANCE LEVEL: SMILING UTTERANCES AND PERCEIVED STANCES}

The smiling behavior, i.e. when and which types of smile are expressed, may determine the perceived stances: both the epistemic stances (the relationship of a person to her talk) and the interpersonal stances (the relationship of a person to her interlocutor). Indeed, a speaker who delivers a verbal message with a smile may be perceived with an amused stance with regard to her speech. Moreover, a smiling person may be perceived as warmer to her interlocutor than a non-smiling one $[4,5]$.

In the domain of virtual characters, some studies have ex- plored the effects of the expressions of smile on the user's perception during an interaction. The results of these studies $[10,7]$ show that smiles of a virtual agents, and in particular the amused smile (compared to the polite smile) enhance its perceived interpersonal stances. However, most of these studies analyze the effects of a smiling virtual characters on the overall interaction. Few research has explored the impact of smiles at an utterance level.

In order to analyze the effects of the expressions of smiles on the user's perception at an utterance level, we have conducted a study to collect the perception the users have of virtual characters when the later deliver a verbal message with or without displaying polite and amused smiles. Videos of virtual characters displaying either both polite and amused smiles or none of these smiles while greeting (polite smile) and telling a joke (amused smile) were created. Users watched these videos and indicated their perception on the following interpersonal stances: spontaneous, stiff, cold, warm, boring, and enjoyable. To measure the effects of smiles on users' perception of the content of the message, we asked users to indicate if the virtual character thinks its riddle funny, if they understood the joke and if they like it. To consider the effect of the appearance on users' perception of virtual character's smiles, we have used two different virtual characters: one male and one female.The results reveal significant effects of both the displayed smiles and of the appearance of the virtual character on the users perception of the interpersonal stances. In line with the results of the studies in Human and Social Sciences [5], the smiles, and in particular the amused smile, enhance the perceived interpersonal stances of the virtual character. The results of our study show also that smile displays by a female virtual character has a higher positive impact on its perceived interpersonal stances than smile displays by a male virtual character. Concerning the epistemic stance, the results show that the expression of the amused smile modifies the perceived virtual character's epistemic stance: users found that the virtual character thinks its riddle is funnier when it expresses an amused smile. The virtual character's smiling behavior may also change users' epistemic stance with regard to the content of the verbal message: users prefer the riddle and judge the riddle funnier when the virtual character expresses both smiles than no smile or only polite smile (for more details on the study see [11]).

In a nutshell, this study shows that the expressions of smile aligned with a verbal message have a significant effect on the perceived epistemic and interpersonal stances of the virtual character, but also on the epistemic stance of the user regarding the content of the message. Moreover, since recent research reveals the positive effects of smiling virtual character on the user's feelings [12], we may suppose that the smiling behavior of the virtual character may change the user's interpersonal stances toward it.

The perceived stances of the virtual characters have been evaluated in a situation in which the user remains passive 
since she is not involved in the conversation with the virtual character (she watches videos of the character). The direct involvement of the user in the interaction may change her perception of the virtual characters' stances.

\section{COMMUNICATIVE LEVEL: PERCEIVED STANCES IN HUMAN-VIRTUAL CHARACTER INTERACTION}

The collected corpus of users' perceptions of virtual characters' stances (Section 3) has been used to develop a computational model of user's potential perception of the virtual character's stance. It endows the virtual character with the capability to estimate the effects of its smiling behavior on the user's perception. Based on this data, we have computed the probabilities of the perceived virtual character's interpersonal stances depending if the virtual character expresses or not an amused or a polite smile in a potentially expected situation. The probabilistic model has been developed with the hypothesis that the specific situation of greeting and of telling a riddle can be extended to other situations in which amused or polite smiles may be expected and expressed ${ }^{1}$. During an interaction between a user and a virtual character, after each sentence pronounced by the virtual character, given its appearance (female or male), its communicative intention, and its smiling behavior (polite smile, amused smile, or no smile), the computational model provides a matrix reflecting the probability of the user's (potential) perception of the virtual character's interpersonal stances (spontaneous, stiff, warm, enjoyable and boring). This model enables the virtual character to measure the potential effects of its smile but also the potential effects of not displaying a specific smile in a situation in which the user may expect this non-verbal behavior (for more details on the model see [11]).

In order to validate this computational model in a general conversation between a user and a virtual character, we have integrated it in the platform SEMAINE, a system that enables users to naturally converse with virtual characters [13]. We have asked 15 participants to interact $3 \mathrm{mn}$ with both, male and female, virtual characters in two conditions: an nonsmiling version in which the virtual characters do not express any smile, and a smiling version in which the virtual characters display amused and polite smiles when they have particular communicative intentions associated to these smiles. After each interaction, we asked users to fill a questionnaire to collect their perception of the virtual characters' interpersonal stances. To evaluate the accuracy of our model to predict the user's perception of the virtual character's interpersonal stances, we have compared the output of our model at the end of the conversation with the responses of the participants to

\footnotetext{
${ }^{1}$ We have supposed that amused and polite smiles may be expected with different communicative intentions. For instance, the intention to communicate satisfaction may come with an amused smile whereas the intention to show agreement may come with a polite smile.
}

the questionnaire. The results partially validate our computational model. The model may be used to predict the potential user's perception of the male virtual character's positive and negative stances but only the perception of the female virtual character's negative stances (the boring and cold stances). It does not enable us to determine the user's perception of the female virtual agent's positive interpersonal stances. The effects of smiles on the perceived female virtual character's stances seem to be too weak to be caught by our model. This phenomena, not noticed in the study at the utterance level, is in line with research in Human and Social Sciences showing that it is the absence of smile (and not the expression) that deteriorates a woman's image [4].

\section{INTERACTIVE LEVEL: DYADIC STANCES IN AN INTERACTION}

During an interaction, "stances are constructed across turns rather than being the product of single turns" [1]. When the epistemic and affective stances of each partner of the interaction are put in presence, dyadic stances can be inferred [14] from diachronic alignment between interactants. For instance, the alignment of the non-verbal behavior of the speaker and of the listener may convey stances of mutual understanding, attention, agreement, interest and pleasantness [15].

In order to evaluate the capacity of virtual characters to convey different dyadic stances through the alignment of their smiles, we have simulated interactions between two virtual characters with different levels of influence of each other's smiles. At a first level, both virtual characters mutually reinforce their smiles (condition 1); that is the smile of agent $\mathrm{A}$ is mimicked by agent $\mathrm{B}$ which in turn influences the smiling behavior of the agent A. Consequently, the smiling behavior of agent $\mathrm{B}$ as response to the smiling behavior of agent A reinforces the smiling behavior of $\mathrm{A}$ in term of duration and intensity, there is a "snowball effect" between the two agents' smiles. At a second level, only agent B reinforces its smiles according to agent A expressed smiles (condition 2). Consequently, agent A is not influenced by agent B's smile. Sixty-six participants viewed video clips of two female agents interacting. Video clips were made for the three conditions. For each video clip, participants were asked to answer questions concerning their perception of the mutual understanding, attention, agreement, interest and pleasantness of the two virtual characters.

The analysis of the results of the study shows that the mutual understanding, attention, interest, agreement and pleasantness are perceived significantly higher when both agents mutually reinforce their smiles according to the other's smiles (condition 1) than when only one agent reinforces its smiles depending on the other's expressed smiles (condition 2). For certain stances, such as the mutual attention and agreement, the smile reinforcement of only one agent (condition 2) seems not to be sufficient. That is, both agents should reinforce their 
smiles to give an impression of mutual attention and agreement [16]. Finally, during an interaction, virtual characters whose smiling behavior dynamically evolves with each other, give the impression to third party to display specific dyadic stances, i.e. stances that is not specific to a virtual character in particular, but that is co-constructed by the two virtual characters put in presence.

\section{CONCLUSION}

In conclusion, the social signal of smile expressed by a virtual character may convey different stances, both epistemic, interpersonal and dyadic. The perception of these different stances depends on the contextual level considered. Indeed, at a signal level, the smile may convey particular emotional (e.g. happiness, embarrassment) or social (e.g. polite) stances. At an utterance level, the smiles may impact both the perceived interpersonal stances and epistemic stances. At a communicative level, the direct involvement of the user has an impact on the perceived stances. The effects of smile expressions on the virtual agent's perceived stances may be different from the utterance level. Finally, at an interaction level, the alignment of smiles between the interactants may convey the particular stances of the dyad to a third party looking at the interaction. Several future works should be considered to identify other factors that may influence perceived stances. Moreover, one of the next steps is to evaluate the dyadic situation between a virtual character and a user; that is we aim to study if a virtual character that reinforces its smiles depending on the expressed smiles of a user may trigger the perception of specific dyadic stances.

Acknowledgments: This work has been financed by the European Projects SSPNet and VERVE.

\section{REFERENCES}

[1] M. Chindamo, J. Allwood, and E. Ahlsen, "Some suggestions for the study of stance in communication," in SocialCom Conference, 2012, pp. 617 -622.

[2] S. F. Kielsing, Stance: Sociolinguistic Perspectives, chapter Style as stance: Stance as the explanation for patterns of sociolinguistic variation, pp. 171-194, Oxford University Press, Oxford, 2009.

[3] M.L. Knapp and J.A. Hall, Nonverbal Communication in Human Interaction, Wadsworth Publishing, 2009.

[4] F.M. Deutsch, D. LeBaron, and M.M. Fryer, "What is in the Smile?," Psychology of Women Quarterly, vol. 11, 1987.

[5] S. Lau, "The effect of smiling on person perception," Journal of Social Psychology, vol. 117, pp. 63-67, 1982.
[6] P. Ekman, Telling Lies: Clues to Deceit in the Marketplace, Politics, and Marriage, Ww norton \& co edition, 2009.

[7] M. Rehm and E. André, "Catch me if you can ? Exploring lying agents in social settings," in International Conference of Autonomous Agents and Multi-Agent Systems (AAMAS). 2005, pp. 937-944, Academic Press Inc.

[8] E. Krumhuber and A. Manstead, "Can Duchenne smiles be feigned? New evidence on felt and false smiles," Emotion, vol. 9, no. 6, pp. 807-820, 2009.

[9] M. Ochs, R. Niewiadomski, P. Brunet, and C. Pelachaud, "Smiling virtual agent in social context," Cognitive Processing, Special Issue on "Social signals. From Theory to Applications”, vol. 13(2), pp. 519-532, 2012.

[10] E. Krumhuber, A. Manstead, and A. Kappas, "Temporal aspects of facial displays in person and expression perception. The effects of smile dynamics, head tilt and gender.," Journal of Nonverbal Behavior, vol. 31, pp. 39-56, 2007.

[11] M. Ochs and C. Pelachaud, "Model of the perception of smiling virtual character," in Proceedings of AAMAS, 2012, pp. 87-94.

[12] N. Krämer, S. Kopp, C. Becker-Asano, and N. Sommer, "Smile and the world will smile with you-the effects of a virtual agent's smile on users' evaluation and behavior," Int. J. Hum.-Comput. Stud., vol. 71, no. 3, pp. 335-349, 2013.

[13] M. Schröder, "The SEMAINE API: Towards a Standards-Based Framework for Building EmotionOriented Systems," Advances in Human-Computer Interaction, vol. vol. 2010, Article ID 319406, pp. 21 pages, 2010.

[14] K. Prepin, M. Ochs, and C. Pelachaud, "Mutual stance building in dyad of virtual agents: Smile alignment and synchronisation," in SocialCom Conference, pp. 938943.

[15] M. Louwerse, R. Dale, E.G. Bard, and P. Jeuniaux, "Behavior matching in multimodal communication is synchronized," Cognitive Science, vol. 36, no. 8, pp. 14041426, 2012.

[16] K. Prepin, M. Ochs, and C. Pelachaud, "Beyond backchannels: co-construction of dyadic stancce by reciprocal reinforcement of smiles between virtual agents," in International Conference CogSci. 